\title{
AN ANALYTICAL APPROACH TO LOW OBSERVABLE MAINTENANCE PRACTICES USING SIMULATION AND MARGINAL ANALYSIS
}

\author{
Stephanie C. Ysebaert \\ Alan W. Johnson \\ John O. Miller \\ Timothy J. Pettit \\ Air Force Institute of Technology \\ Department of Operational Sciences \\ 2950 Hobson Way, Bldg 641 \\ Wright-Patterson AFB, OH 45433, USA
}

\begin{abstract}
The F-22 Raptor is a unique aircraft with many technological advantages and superior capabilities. The aircraft's stealth capability is a function of many design aspects, including coatings that cover the outside of the aircraft and help mitigate radar detection. Maintaining these Low Observable coatings has its own set of challenges to include an inexperienced work force, time consuming procedures, and demanding maneuvers of a fifth generation fighter aircraft. Another challenge facing the F-22 fleet is low aircraft availability, where the aircraft is down for numerous reasons. Using a simulation built in ARENA, process improvements to Low Observable maintenance can be quantified with a goal of improving aircraft availability. One example of process improvements, the use of extra stock panels is tested in the simulation to see the potential marginal improvement to Aircraft Availability.
\end{abstract}

\section{THE AIRCRAFT: F-22 RAPTOR}

Designed as a fifth generation fighter aircraft and a member of the Global Strike Task Force, the F-22 is capable of air to air and air to ground missions. The aircraft's stealth capability, known as Low Observable (LO) is essential to maintaining its superiority. Proper maintenance of the stealth capability insures the Raptor will avoid radar detection and can perform all its varied capabilities (Hill 2010). The Raptor is stationed at a variety of locations which adds to the complexity of aircraft maintenance.

\subsection{Aircraft Availability}

Aircraft Availability is a metric that assesses the readiness of a wing or fleet to meet its stated mission. In its simplest terms, aircraft availability can be defined as the ratio of time available to do a mission to total time a unit possesses the aircraft. Equation 1 shows the mathematical formula for calculating availability.

$$
A_{0}=\frac{T A I-N M C M-N M C S-N M C B-U P N R-D E P O T}{T A I}=\frac{M C}{T A I}
$$

In Equation 1, all units are in hours. TAI or Total Active Inventory is the number of hours the fleet of aircraft can perform its mission. The next five variables represent the hours an aircraft is not available to complete its mission because it is in one of five maintenance statuses. The five maintenance statuses shown are Non-Mission Capable due to Maintenance (NMCM), Non-Mission Capable due to Supply 
(NMCS), Non-Mission Capable due to Both Maintenance and Supply (NMCB), Unit Possessed NonReporting (UPNR), and Depot Level Maintenance (DEPOT). This research focuses on Non-Mission Capable due to Maintenance (NMCM), which accounts for approximately $22 \%$ of the aircraft's total active hours. Low Observable System Maintenance is a small subset of NMCM, less than a quarter of NMCM hours. Thus, improvements to aircraft availability through Low Observable maintenance practices will be small, but significant none the less.

\section{PROBLEM DEFINITION}

One example of improved maintenance processes is the idea of using extra supply of panels. Certain panels are available to replace damaged panels and return the aircraft to its mission in a fraction of the time compared to previous methods with no extra panels. The damaged panel is then repaired offline in a work area where it will become extra supply for the next damaged panel. Using Subject Matter Expert opinions, 16 panels were chosen as extra panel candidates and grouped into six panel types based on similar repair distributions.

When a panel is converted to extra supply panel, no additional manpower or resources are required that was not being used when the panel was not extra supply. The decision of when to repair the damaged panel is not affected. By having extra supply panels there is now another option. The work to repair the damaged panel is still performed; however, the aircraft is no longer down waiting for panel repair. Extra supply of panels allow the aircraft to return to Mission Capable status quicker by removing the time to restore the panel from the aircraft's critical path. Subject Matter Experts believe when an extra supply panel is used, $10 \%$ of previous maintenance time is still required to account for physically removing and replacing the panel.

\section{LITERATURE REVIEW}

\subsection{Simulation}

Given the complex nature of Low Observable aircraft maintenance and the need for sensitivity analysis, a simulation was chosen to model the extra supply panel process. As a problem solving tool simulations can be very powerful. Although a model is only an abstraction of reality, there are many benefits of using a simulation for analysis. Modeling a problem allows for exploration of key variables and processes without costly commitments or repercussions. Simulations also allow for visualization and analysis of complex systems too intricate for mathematical equations or direct experimentation (Banks et al. 2005). Montevechi and Pinho (2007) suggest that simulations are a useful tool because they offer automation, replication and higher comprehension at a fraction of the cost of actually implementing proposed changes. A limitation to using simulation is the tradeoff between model accuracy and the confidence in observations and analysis. The power of subsequent analysis stems from the strength of the model itself. The process of assessing the strength or adequacy of a model is known as Verification and Validation (V\&V). Jordan, Melouk, and Faas (2009) discuss the importance of V\&V in simulation and offer some common challenges to the process. They note that $\mathrm{V} \& \mathrm{~V}$ is time consuming, can be costly, is difficult, complex, and requires more data than sometimes is available. Despite the disadvantages, model builders agree that $\mathrm{V} \& \mathrm{~V}$ is important and often use subject matter experts and personnel in the shop to check the model structure and data input. Another way a model is verified for robustness is by varying the inputs across a range and checking the output for trends in the correct direction. For example, if personnel were decreased in the model, one would expect wait time of entities to increase. This confirmation allows for greater confidence in the results presented.

\subsection{Simulations in Aircraft Maintenance and Sortie Generation}

Lessons were also learned from similar simulations in aircraft maintenance. The United States Air Force uses a variety of simulation analyses, employing models such as LCOM (Logistics Composite Model), 
SIMFORCE (Scalable Integration Model for Objective Resource Capability) and LogSAM (Logistics Simulation and Analysis Model). Boyle (1990) discusses LCOM in-depth while Faas (2003) gives a overview of all three Air Force Models. SIMFORCE is built in ARENA and could be of potential use for future research in Low Observable maintenance practices as a desktop tool for decision makers. Although other capable programs are available, each of these models has varying levels of complexity and limited adaptability and was not chosen to implement this research.

Faas (2003), Iakovidis (2005), and Parks (2010) successfully conducted aircraft maintenance simulations in ARENA. This suggests the software is credible and capable of what we want to model. A similar approach was also taken by the authors mentioned above by simulating aircraft as entities flowing through the model, and keeping track of key details to trigger flow through certain maintenance processes. The authors found success using similar simulation structures, with each section of the model having a unique function, and all sections then tied together through logic gates. This model building technique allows for stepwise model construction and greater confidence in an accurate model.

\subsection{Designed Experiment and Marginal Analysis}

Design of Experiments (DOE) is the procedure of systematically testing design factors (inputs) at varying levels to discover if a factor is important, or has a significant effect on the output of interest. As described by Kelton (1999), "One of the principal goals of experimental design is to estimate how changes in input factors affect the results or responses, of the experiment." In general, DOE can be used to screen which factors are important or to predict optimal factor combinations. DOE is used primarily in real life (physical) systems, but can also be applied to deterministic and stochastic simulation models (Kleijnen 2008).

There are many advantages of using experimental design in simulations as demonstrated in articles by Kelton (1999), Kleijnen, Sanchez, and Lucas (2005), Kleijnen (2008), Montevechi and Pinho (2007), and Sanchez (2006). Montevechi and Pinho (2007) show that there are many advantages to combining these two different techniques, to include "avoiding trial and error to seek solutions" and "improving the performance on the simulation process," Kleijnen, Sanchez, and Lucas (2005) asserts that the two methods rarely combine because "research related to design of experiments is frequently found in specialized magazines, rarely read by simulation practitioners." Knowing that a simulation is a model of the real world, one can think of it as a black box "that implicitly transforms inputs (such as factor-level settings) into outputs" (Kleijnen, Sanchez, and Lucas 2005). The designed experiment of interest can be represented as $R V=f(I V)$, where the response variable $(R V)$ or output of the experiment is some function of the input variables $(I V)$ or factors. The transformation $f$ represents the simulation itself (Kelton 1999).

There are also many advantages of using simulation as the transformation from input to output, as opposed to an operational test. One advantage is the control over all input variables. Error is greatly reduced because of the control over all the factors (Montevechi and Pinho 2007). Another advantage is the ease of replication in a simulated environment. Many replications of the model lead to more accurate estimations of the interactions and main effects, as well as narrower confidence intervals.

One specific designed experiment known as the "shopping list" approach was used in determining which panels to acquire based on a marginal, or per unit, increase to availability. This approach is based on the Aircraft Sustainability Model (ASM), created by the LMI Corporation for the United States Air Force.

The ASM is a "mathematical model that computes an optimal spares mix to support a wide range of possible operating scenarios" (Slay et al. 1996). The ASM gives a "shopping list" of spare parts that should be purchased based on a user defined metric such as availability, subject to a constrained budget. A benefit to cost ratio should be maximized when choosing which parts to have in supply. Shown in the ASM Report, Figure 1 depicts the solution curve, where availability or budget can be specified, and the other variable is then determined. 


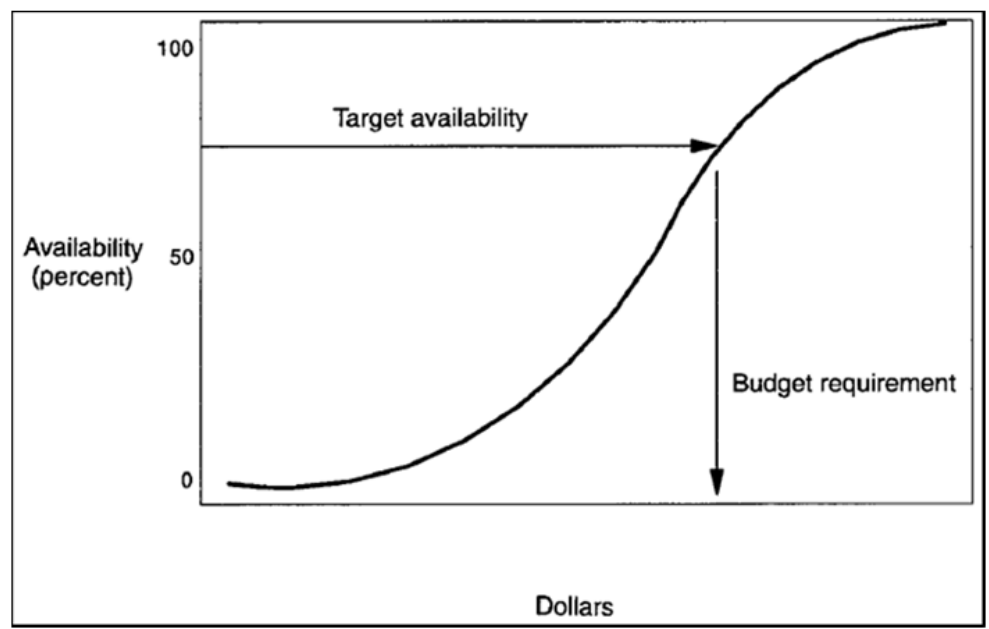

Figure 1: Aircraft Sustainability Model shopping list curve

\section{METHODOLOGY}

\subsection{Conceptual Model}

To offer insight into Low Observable maintenance and specifically the benefit of extra supply panels, a simulation model was created in ARENA. This discrete event simulation was necessary to capture the complex nature of maintenance processes and to simulate the accrual of damages to the aircraft's coatings. Throughout the model building process, subject matter experts were consulted to offer their experience and give estimates when actual data was not available.

The conceptual model for the simulation is shown in Figure 2. First, aircraft are created then checked against a schedule to see if any planned maintenance actions are required. If so, those aircraft are removed from the pool of available aircraft.

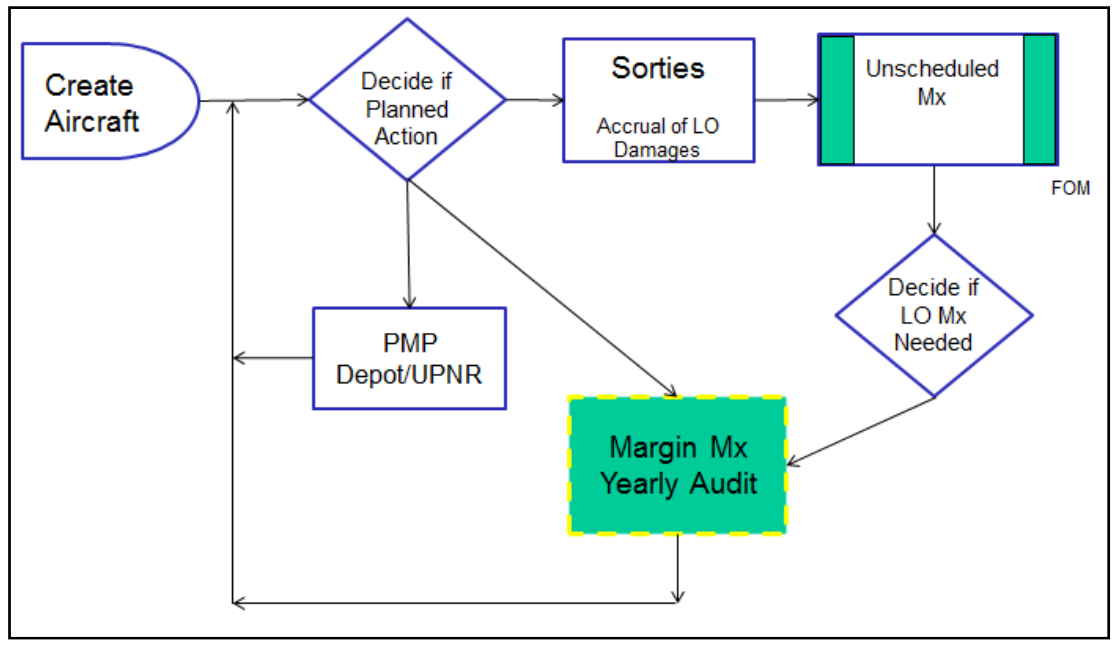

Figure 2: Conceptual simulation model

Next, the available aircraft fly a sortie, followed by any unscheduled maintenance. Then, Low Observable Maintenance might be needed, after which the cycle repeats. The shaded regions in Figure 2 represent the times when Low Observable maintenance is accomplished. The simulation mirrors one 
squadron of 20 aircraft. The entities flow through six sub models where planned actions are updated, sorties are flown and regular and Low Observable maintenance occurs. The entities are created once and never leave the model, they are only delayed at certain times to simulate real world base operations.

\subsection{Model Verification and Validation}

Model verification is the process of creating a functional model, free of errors. To accomplish this task the model was built in a piece-wise fashion, building one sub model at a time and using output data written to spreadsheet files to verify correct scenarios. Also, low level detail was modeled first to insure correct logic, followed by modeling more detailed processes once the foundation was correct.

The model was also validated by consulting with experts throughout its creation for accurate data and decisions modeled. Although particular values are not releasable in this paper, we found that simulated sortie generation rates, yearly audits, and depot maintenance frequency all compared well to actual values. Damage Type and Frequency was also validated by expert opinion. Status hours for the fleet and each base were provided for select data entry as well as for validation purposes.

\subsection{Executing a Designed Experiment-Marginal Analysis}

Once the model was constructed, properly verified, and validated, the next step was to vary inputs and see how they affect the associated simulation output.

Aircraft availability is the primary output, defined as the ratio of available hours to possessed hours. In the simulation, the total active inventory (TAI) consists of 20 aircraft, with 30 days per month time period. Panel Hours is a secondary output of the model and is the summation of the time each aircraft spends in the panel sub models, waiting for or receiving panel maintenance. To measure the effect of extra supply panels, each panel type's quantity is varied and the change in output metric is recorded. The six variables of interest are the resource quantity of panel types 1- 6. The quantity of extra panels is varied over categorical levels $0,1,2 \ldots n$. Zero panels represent current operations with no extra supply panels available as spares.

To realize the greatest improvement to aircraft availability, ARENA's Process Analyzer was used to vary the six controls against the output responses: availability and panel hours. Availability was calculated as the one year average after a six month warm up of fifty replications. Fifty replications were used because it helped reduce variability seen in the output variables.

Next, a "shopping list" approach was taken to realize the marginal improvement to availability as one additional panel was purchased, or selected. Groups of six runs were executed and the panel with the highest increase in availability compared to the previous panel scenario is chosen. To begin, a scenario is created where only one quantity of each panel resource exists in the model. The scenario is run and the panel resource that achieves highest availability is chosen to be on the list. Next, an additional quantity of the chosen panel is added, and again the scenario is run. The scenario with the best availability with two panels is selected, and the second panel that created this pair is added to the list. This process continues until no more improvement is seen, or a budget runs out of money. The use of "per-unit input increasing output" logic answers the question, "If I could only purchase one more panel, which one would it be to increase availability the most?" This process repeats until no more substantial increase in availability is observed, or until the budget for panels is exceeded.

\section{RESULTS AND ANALYSIS}

Given the nature of this non-terminating simulation, our first objective is to find a point in time where the output leveled out and entered the steady state. Fifty replications each ten years long were used for trial runs. In these runs the baseline model was executed, with no extra supply panels. The transient period was identified at six months, as seen in Figure 3. 
Figure 3 shows that there is some cyclical nature to the output and this behavior was retained in the steady state nature of the model. Availability increases around the 50 month and 110 month marks. This could be attributed to the periodic nature of aircraft maintenance.

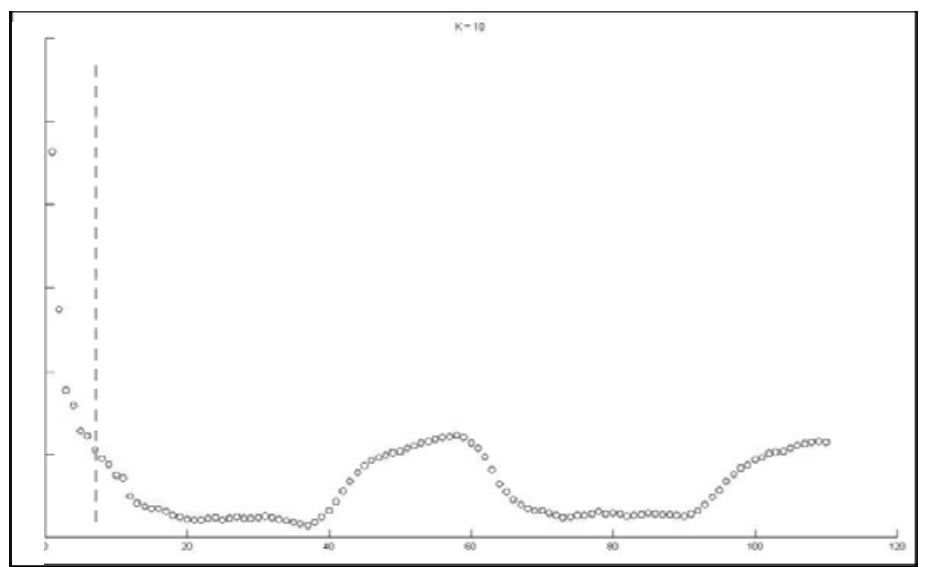

Figure 3: Transient conditions analysis - Abscissa scale is in months, and ordinate represents availability

\subsection{Test Panel Types for Marginal Improvement to Availability}

Using a warm up period of six months and a monthly average for one year, groups of panels were run using the "shopping list" approach, executed with Process Analyzer. The best panel combination that increases availability is seen from Panel 3, then Panel 2, Panel 5, Panel 1, Panel 4, and Panel 4. Availability increased $1.2 \%$ with six panels over the base case of zero panels. Although overall availability is increasing, the confidence intervals between subsequent panels overlap suggesting that the increase is not statistically significant. To further explore this idea, Paired- $t$ tests were conducted with each pair-wise combination of panels. Paired- $t$ tests were used because Common Random Numbers were employed in the model, synchronizing the model replications and reducing variability. Paired- $t$ tests compare the mean difference between scenarios to the hypothesized mean difference of zero, or no difference. Because the mean difference of the outputs is now considered independent and identically distributed, a $t$-statistic can be used to compare additional panels to the base case of zero panels. The test gathers strength of evidence for the alternative hypothesis in the form of a $p$-value, that the mean difference is not equal to zero. Small $p$-values support the case for rejection of the null hypothesis in favor of the alternative. Table 1 shows the $p$-values for the comparisons between the seven scenarios, with the bold face denoting significant "shopping lists".

Table 1: $p$-value of all pair-wise comparison of additional panels

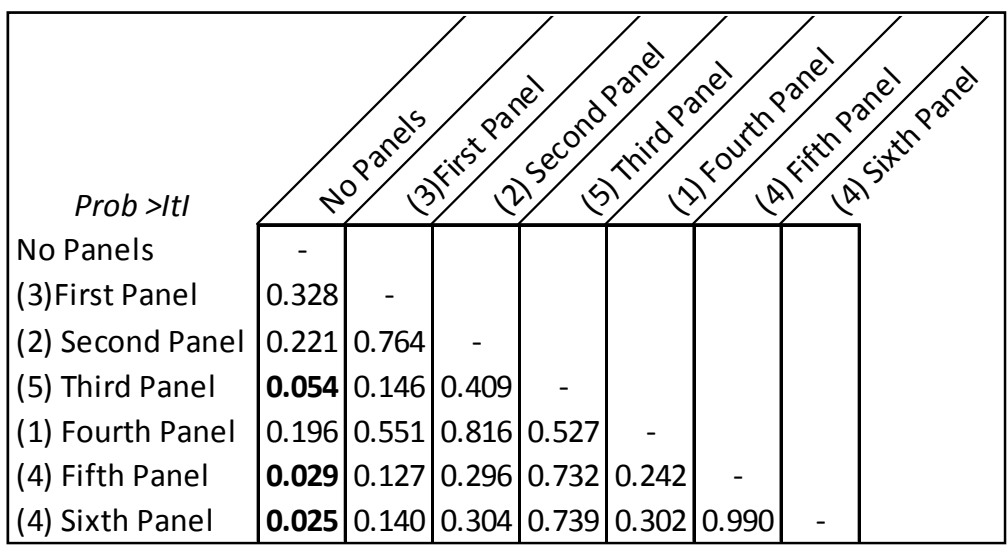


For example, acquiring one-each of the respective Type 3, Type 2, and Type 5 panels produces an increase in availability over the base case of zero panels, at significance $p=0.054$. However, acquiring these three panels is not statistically better $(p=0.409)$ than acquiring only the first two. Of interest is that subsequent panels never show a marginally significant increase in availability (as seen by large $p$-values on Table 1's main diagonal), but adding the fifth and sixth panels both produce respective availability increases over the base scenario of zero panels. Also of interest is the finding that while adding the first three and then a respective fifth and six panel is statistically desirable compared to the base case, we observe that adding the fourth panel is not $(p=0.196)$ ! We suspect this anomaly is due to "random bounce." The overall effect of panel acquisition increases to aircraft availability might not be statistically significant, but the panel additions may be practically significant for the maintenance time saved by using the extra supply panels. The $1.2 \%$ effect on availability is not profound due to randomness and other maintenance events that an aircraft encounters in the model.

\subsection{Test Panel Types for Marginal Improvement to Panel Hours}

A second output of interest, Panel Hours was captured at the same time the experiment was run for Availability. Panel Hours is the summation of maintenance time the aircraft spend in a back shop receiving a new panel or waiting for a repaired or spare part panel. The less time an aircraft spends in non-mission capable status in the back shop, the more time an aircraft can perform its mission. As additional panels are acquired, Panel hours decrease, but the time saved may not translate completely to increased availability. In an actual flying unit, human scheduling can provide mitigating actions to help translate panel hours to higher aircraft availability and represents a limitation of our models.

Using the "shopping list" approach, six different panels were selected to decrease panel hours. Using six panels saves on average 400 hours over the scenario with zero panels in extra supply. After acquiring six panels, the decrease to panel hours was no longer significant.

\subsection{Cost Benefit Analysis}

Another way of determining the best shopping list of panels would be to compare the marginal availability contribution per dollar cost. The assumption is made that the panel types are constructed and stored similarly. The same methodology was used to construct the shopping list, however the output of interest is the change in availability per cost. Cost is calculated as a function of the size of the panel and $25 \%$ carrying costs for storage and security.

Thus, an additional shopping list was created using the "shopping list" approach and panels were selected based on the greatest change in Availability to Cost ratio. The best panels in decreasing order of ratio improvement are Panel 3, Panel 2, Panel 5, Panel 1, Panel 4, which is the same selection found when comparing availability alone. Similarly, panels could be selected based on the change in Panel Hours to Cost ratio. This analysis resulted in a different selection of panels for the shopping list. Table 2 shows the three main goals, their respective shopping list of panels, and the change to availability and panel hours associated with that purchase.

Table 2: Summary of shopping list goals

\begin{tabular}{|lccc|}
\hline \multicolumn{1}{|c}{ Goal } & Shopping List & $\begin{array}{c}\text { Availability } \\
\text { Increase }\end{array}$ & $\begin{array}{c}\text { Panel Hour } \\
\text { Decrease }\end{array}$ \\
\hline Max Availability & $3-2-5-1-4-4$ & $1.2 \%$ & 345 \\
Min Panel Hours & $1-3-2-6-5-4$ & $1.0 \%$ & 400 \\
Max Cost Benefit (PH) & $6-2-4-6-5-3$ & $0.4 \%$ & 261 \\
\hline
\end{tabular}

The point estimate of the mean panel hours as each additional panel is selected is shown in Figure 4. The minimum panel hour scenario (blue diamond symbol) reduces panel hours the most but has the high- 
est cost. The cost efficient scenario (green triangle symbol) resulted in the highest return on investment at $\$ 6 \mathrm{M}$ and reduces panel hours by 261 hours. Thus, different scenarios can be selected based on a specific goal. Ultimately, there is a tradeoff between higher availability or lower panel hours and increased cost.

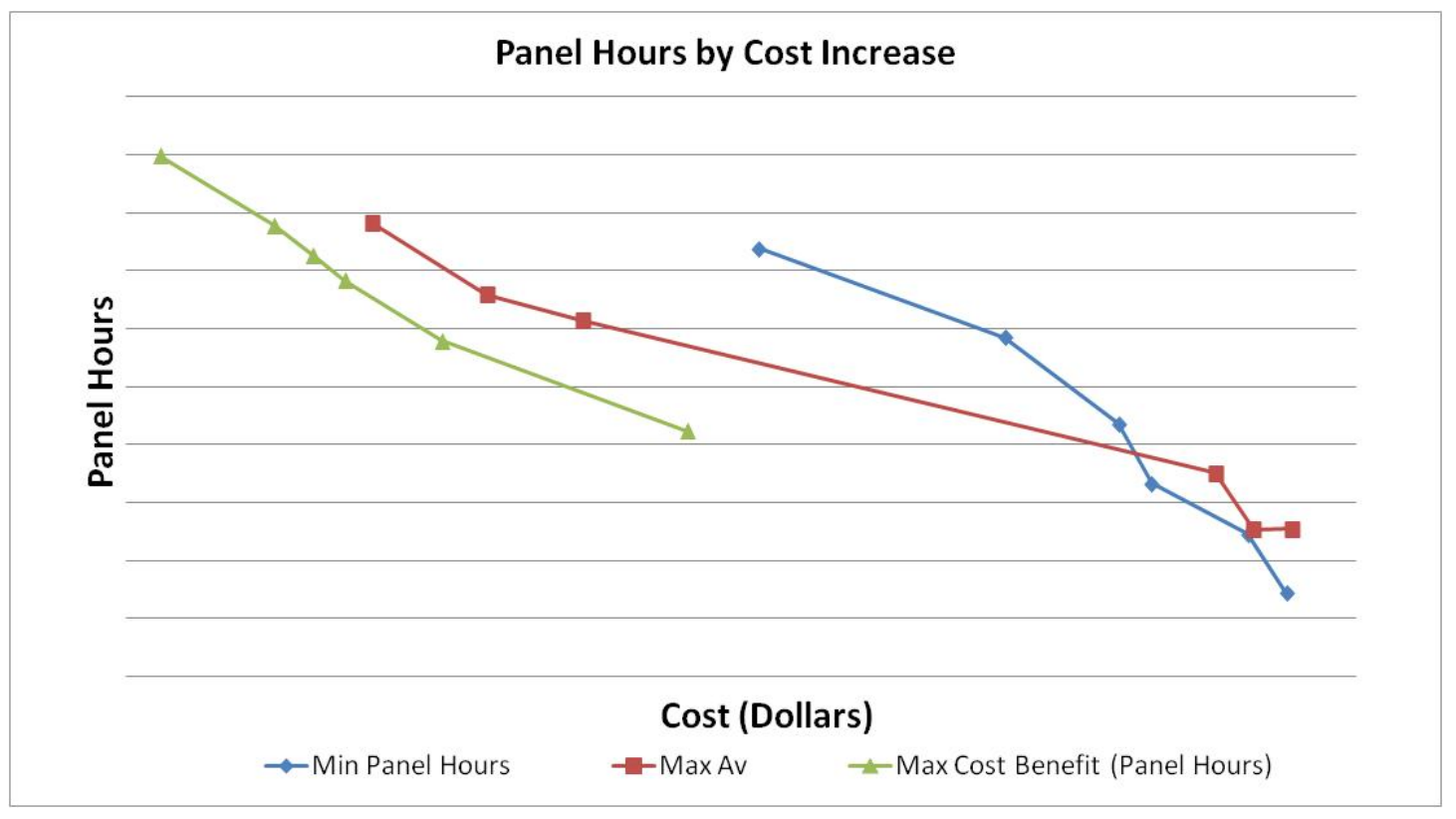

Figure 4: Shopping list comparison

\section{CONCLUSION}

This research estimates the impact on aircraft availability by stocking extra supply panels for F-22 aircraft. However, not every panel offers the same return for increased availability or decreased panel hours. Panel Type 1 offers the most time benefit, while Panel Type 3 offers the best availability increase. We believe in practice that the time saved using Panel Type 1 could be better utilized, and would increase availability the most. Also, there is a limit to how much availability can improve from using extra supply panels. After five or six panels are purchased, availability must be improved by other means. Due to the small scope of Low Observable maintenance compared to the larger maintenance picture, improvement to availability was expected to be small. However, aircraft wait time saved in the panel shops was significant.

As a recommendation for future work, an area of improvement could be to arrange for aircraft maintenance personnel to record additional information on repair types and durations. Our available data was limited: the panel frequency and durations are estimates and could offer clearer analysis if future data is proven to be more accurate. Also, research could be helpful in determining an optimal scheduling function for the F-22 fleet. Treating Low Observable as a subsystem that must be managed up front instead of the current reactive approaches could allow better aircraft utilization. A scheduling tool could allow for more opportunistic maintenance, whether for Low Observable or regular maintenance. There is a balance between maintenance and operations, and a more analytical schedule could offer consistency between maintenance units and remove the manual input factor that is currently used.

We found that marginal analysis provides a simple, intuitive designed experiment approach for simulation-driven inventory estimation, but its application seems under-represented in the literature. Hopefully this paper will help stimulate more widespread adoption. 
Ysebaert, Johnson, Miller, and Pettit

\section{REFERENCES}

Banks, J., J. S. Carson, B. L. Nelson, and D. M. Nicol. 2005. Discrete-Event System Simulation. 4th ed. Upper Saddle River, New Jersey: Prentice-Hall, Inc.

Boyle, E. 1990. “LCOM Explained”. Report no. ARHRL-TP90-58. Logistics and Human Factors Division. Defense Technical Information Center, ADA 224497.

Faas, P. D. 2003. "Simulation of Autonomic Logistics System (ASL) Sortie Generation." Master's Thesis. Air Force Institute of Technology.

Hill, D. 2010. "LO Technicians Keep Raptors 'Off the Radar'." Joint Base Langley-Eustis. Air Force $\begin{array}{lllllll}\text { Print News. } 21 & \text { June } 2010 . & \text { Accessed }\end{array}$ http://www.jble.af.mil/news/story.asp?id=123210194.

Iakovidis, K. 2005. “Comparing F-16 Maintenance Scheduling Philosophies.” Master's Thesis. Air Force Institute of Technology.

Jordan, J. D., S. H. Melouk, and P. D. Faas. 2009. "Analyzing Production Modifications of a C-130 Engine Repair Facility Using Simulation", In Proceedings of the 2009 Winter Simulation Conference, edited by M. D. Rosetti, R. R. Hill, B. Johansson, A. Dunkin, and R. G. Ingals, 1770-1777. Piscataway, New Jersey: Institute of Electrical and Electronics Engineers, Inc.

Kelton, W. D. 1999. "Designing Simulation Experiments." In Proceedings of the 1999 Winter Simulation Conference, edited by P. A. Farrington, H. B. Nembhard, D. T. Sturrock, and G. W. Evans, 33-38. Piscataway, New Jersey: Institute of Electrical and Electronics Engineers, Inc.

Kleijnen J. P., S. M. Sanchez, and T. W. Lucas. 2005. "State-of-the-Art Review: A User's Guide to the Brave New World of Designing Simulation Experiments."- INFORMS Journal on Computing 17 (3): 263.

Kleijnen, J. P.C. 2008. "Design of Experiments: Overview." In Proceedings of the 2008 Winter Simulation Conference, edited by S. J Mason, R. R. Hill, L. Monch, O. Rose, T. Jefferson, J. W., 479-488. Piscataway, New Jersey: Institute of Electrical and Electronics Engineers, Inc.

Montevechi, J. A. B., and A. F. Pinho. 2007."Application of Design of Experiments on the Simulation of a Process in Automotive Industry." In Proceedings of the 2007 Winter Simulation Conference, edited by S. G. Henderson, B. Biller, M. H. Hsieh, J. Shortle, J. D. Tew, and R. R. Barton, 1601-1609. Piscataway, New Jersey: Institute of Electrical and Electronics Engineers, Inc.

Park, A. R. 2010. "Simulation Analysis of High Velocity Maintenance for the B-1B." Master's Thesis. Air Force Institute of Technology.

Sanchez, S. M. 2006. "Work Smarter Not Harder: Guidelines for Designing Simulation Experiments." In Proceedings of the 2006 Winter Simulation Conference, edited by L. F. Perrone, F. P. Wieland, J. Liu, B. G. Lawson, D. M. Nicol, and R. M. Fujimoto, 47-57. Piscataway, New Jersey: Institute of Electrical and Electronics Engineers, Inc.

Slay, F. M., T. C. Bachman, R. C. Kline, T. J. O'Malley, and F. L. Eichorn. 1996. “Optimizing Spares Support: The Aircraft Sustainability Model.” Report no. LMI-AF501MR1. National Technical Information Center: AD-A320 502/8.

\section{AUTHOR BIOGRAPHIES}

STEPHANIE C. YSEBAERT is an operations research analyst and First Lieutenant in the United States Air Force currently working in the Human Performance Wing, Air Force Research Lab at Wright Patterson AFB, OH. She received her M.S. in Operations Research from the Air Force Institute of Technology in 2011. She can be reached by emailing at stephanie.ysebaert@us.af.mil.

ALAN W. JOHNSON is an associate professor in the Department of Operational Sciences at the Air Force Institute of Technology (AFIT). He earned a B.S. in Mechanical Engineering from Montana State University, M.S. in Engineering Management from the Air Force Institute of Technology, and Ph.D. in Industrial Engineering from Virginia Tech. His research interests include all aspects of military logistics, 
but emphasize reliability and maintainability and the logistics support aspects of space flight systems. He is a retired Air Force officer. His email address is alan.johnson@afit.edu.

JOHN O. MILLER is a 1980 graduate of the U.S. Air Force Academy (USAFA) and retired from the Air Force as a Lt. Colonel in January 2003. In addition to his undergraduate degree from USAFA, he received an M.B.A. from the University of Missouri at Columbia in 1983, his M.S. in Operations Research from the Air Force Institute of Technology (AFIT) in 1987, and his Ph.D. in Industrial Engineering from The Ohio State University in 1997. Dr. Miller is an associate professor in the Department of Operational Sciences at AFIT. His research interests include combat modeling, computer simulation, and ranking and selection. His email address is jmiller@afit.edu.

TIMOTHY J. PETTIT is an assistant professor of management at the U.S. Air Force Academy, Colorado, while on active duty as a Lieutenant Colonel. In addition to his continuing research in commercial and military supply chain resilience, Lieutenant Colonel Pettit teaches Operations Management and Supply Chain Management. His U.S. Air Force career includes a breadth of logistics experience serving as an aircraft maintenance officer and a logistics readiness officer, leading F-16, A-10, and F-15 maintenance organizations in-garrison and deployed. He has published in the Air Force Journal of Logistics and the Supply Chain and Logistics Journal. Lieutenant Colonel Pettit earned a B.S. from Iowa State University in Aerospace Engineering, M.S. in Logistics Management from the Air Force Institute of Technology, and Ph.D. in Business Administration from The Ohio State University. His email address is Timothy.Pettit@usafa.edu. 